\title{
The Platformization of Gender and Sexual Identities: An Algorithmic Analysis of Pornhub
}

Ilir Rama (ilir.rama@unimi.it), University of Milan

Lucia Bainotti (lucia.bainotti@unito.it), University of Amsterdam

Alessandro Gandini (alessandro.gandini@unimi.it), University of Milan

Giulia Giorgi (giulia.giorgi@unito.it), University of Milan

Silvia Semenzin (silvia.semenzin@ucm.es), Complutense University of Madrid

Claudio Agosti (claudio@tracking.exposed), Tracking Exposed

Giulia Corona (giulia@tracking.exposed), Tracking Exposed

Salvatore Romano (salvatore@tracking.exposed), Tracking Exposed

Online pornography, like other forms of cultural production, is increasingly subject to processes of platformization. While research has focused on the diffusion of online pornography and its broader implications, less attention has been paid to the algorithmic infrastructures through which platforms distribute and manage pornographic content, and how this might reiterate socially embedded views and perspectives. To fill this gap, we consider how Pornhub, currently the leading porn platform, establishes the gender identity of its users, and how this affects the structure of the platform and the distribution and recommendation of content within it. We collected data about 1.600 variations of Pornhub's homepages, as well as data about 25.000 videos suggested to 10 accounts with differing self-declared gender identities. Through this data and an analysis of the 
signup procedure, we underline how Pornhub segments, distributes, and manages content based on the profiling of its users, increasingly following the logics of the platformization of content. Findings point to how Pornhub's algorithmic suggestions and the structure of the platform concur to reiterate a heteronormative perspective on sexual desire, sexuality, and gender identities.

Keywords: Online pornography; Pornhub; heteronormativity; digital platforms; gender; platformization; algorithms.

This article has been accepted for publication in Porn Studies, published by Taylor \& Francis. 


\section{Introduction}

Online pornography is an increasingly pervasive phenomenon, that influences sexual and affective practices (Albury 2014) and frames gender exploration and performativity (Scarcelli 2015). Popular pornographic websites, such as Pornhub and xHamster, are deemed to reinforce a male, white and heterosexual point of view, and thus contribute to foster hegemonic masculinity (Burke 2016), the sexualization of minorities (Fritz et al. 2020), and a heteronormative porn culture (Saunders 2020). However, how exactly this happens has remained largely overlooked. At a platform level, the role of algorithms is pivotal in these processes. Algorithms contribute to manage content visibility (Bucher 2012) and can reiterate the gender bias coded into them (Noble 2018), reifying a specific view of the world due to their social embeddedness (Pasquale 2016). Existing research already points out the relevance of algorithms in relation to pornography, specifically with reference to pornography detection (Gehl, Moyer-Horner, and Yeo 2017), content moderation (Gerrard \& Thornham 2020), and the creation of pornographic content by means of AI (van der Nagel 2020). Yet, limited attention has so far been paid to the role played by algorithmic recommendations in reiterating heteronormative perspectives on gender, sexual interests and practices on digital platforms.

To fill this gap, this article analyses data collected from one of the biggest porn streaming platforms, PornHub, with the aim of inquiring how its recommendation system might vary according to specific socio-demographic characteristics. To empirically account for PornHub's recommendation system, we take advantage of the browser extension 'Pornhub Tracking Exposed' (poTREX), which aims to collect evidence on profiling. The data produced allows researching how user profiling affects the distribution of content, and reveals the economic logics underpinning the platform (Agosti and Corona 2020; Raustiala and Sprigman 2018). In so doing, we consider 
how the recommendation system changes according to users' self-declared gender and sexual interests: this includes changes in categories of the homepage layout, such as recommended videos, suggested categories, and popular content. Using a set of automated user accounts, we recreate the browsing activity of users of the website on the basis of predetermined viewing patterns across two dimensions: gender identity and sexual interests, assigned by the researchers (Sandvig 2014). Results are then analysed focusing on the affordances of the platform such as categories, accounts (e.g., verified, channel, user), rating, and views, to assess the extent to which recommended content varies according to self-disclosed information.

Based on this analysis, we argue that the combination of platform affordances and algorithmic suggestions on Pornhub significantly contributes to a heteronormative perspective on sexual desire and sexuality typical of a heterosexual, white, and hegemonic masculinity. Data show how the platform leverages on affordances to build fixed and limited user gender identities; these work in conjunction with its algorithmic infrastructure to distribute content, segment audiences, and recommend personalized videos. We argue that these mechanisms, which constitute the backbone of the website, contribute to reify and reiterate the heteronormative biases embedded into them. Such an understanding of gender identities and sexuality, as driven by the political economy of the platform, has serious implications for how it can reproduce and foster hegemonic values and views to the potential detriment of minoritarian identities.

The article is structured as follows. In the next section we contextualize our research and discuss how it is in dialogue with both relevant existing studies on pornography and heteronormativity and the broader logics of platformization of cultural production (Nieborg and Poell 2018). Subsequently, we illustrate the methodological steps we have taken to undertake our data collection and then present the main findings that emerge from our analysis. In the conclusive 
section we reflect on how our contribution brings forward important implications for future research at the crossroads of critical porn studies and the platform economy.

\section{Theoretical framework}

\section{Online Pornography and Heteronormativity}

The development of the web has allowed for a large amount of pornographic material to circulate and be accessed by a variety of new publics, which deserve further attention as they defy simple categorizations based on national borders or general publics (Paasonen, 2009:51). Today, the digital porn industry is a lively sector that attracts more users than Twitter, Netflix and Amazon combined (Saunders 2020). According to existing research, 15\% of all websites are pornographic and $20 \%$ of all searches on mobile devices across the world are for pornography (Barrie 2017). In other words, the porn industry has come to represent one of the most rentable sectors of digital capitalism (Saunders 2020, 3).

Arguably, the rise of online pornography has deeply changed the genre, seeing an enormous growth of self-produced material and an increase in competition. The pornographic industry was a pioneer in shifting consumption to prosumption (Ritzer 2010). According to Adam Arvidsson (2017, 74), 'the Internet realizes the hidden potential of the masturbatory economy', as pornography consumers become subjects capable of producing value in line with the information economy (Arvidsson 2007). One of the consequences of these changes in contemporary pornography is that the industry has become more concerned with 'representing, conceptualizing and celebrating sex as a form of labour', instead of representing sexual pleasure (Saunders 2020, 
4), thus also leaving room for more extreme, violent and radical forms of representing sex. In fact, with the advent of digital capitalism, obscenity, excess and subversion have been reappropriated by the pornographic industry as 'vital bases for digital forms of economisation' (Saunders 2020, $5)$.

In this context, online pornography has also benefitted from various positive changes, such as the shift from a predominantly male audience to diverse publics (Ashton et al. 2019), the emergence of a variety of sexual interests (Maziéres et al. 2014), and the rise of communities usually considered illegitimate (Maris et al. 2020). Nevertheless, online pornography maintains some critical issues, especially the fact that such forms of pornography tend to reproduce the male gaze and to foster hegemonic masculinity practices (Saunders 2020; Burke 2016). While pornographic websites concur to reinforce a heterosexual and male point of view, thus contributing to fuel a persistent heteronormative porn culture (Saunders 2020), efforts to the determine how this takes place have been scarce.

Recently, heteronormativity has been researched through the lens of categorization and representation. Saunders (2020) extensively analyses the relation between datafication processes and the necessity of ordering, listing and classifying porn content. Pornographic websites are built on the basis of categorizing bodies, races and sexual behaviors; these classifications are predominantly concerned with labelling women and categorizing female bodies from a male and heterosexual point of view. Very rarely, in fact, heterosexual porn websites offer categories that anatomise and classify the male body. When this happens, it is generally in a racialized way, such as the "Big Black Cock" category. Furthermore, sex performers are visibly listed on porn websites mostly when they are females, whereas a similar categorization is not as spread for male performers (Saunders 2020,71). She shows that sexual classifications on pornographic websites, 
as well as the specific ways non-heteronormative sexualities are classified, depict and construct women and non-white, non-heterosexual or differently able people as 'deviant others' (Saunders 2020, 70). Such classifications flatten and minimize the spectrum of gender and sexuality, thus reproducing heteronormativity. Saunders also considers the category 'lesbian', in which female homosexuality is categorized according to heteronormative labels and reduced only to the satisfaction of the male gaze. Rather than homosexual intercourse, then, lesbian sex is often depicted as a trivial play among women friends. Moreover, heteronormativity is often linked to misogynist discriminations, for the way women are labelled on the databases of mainstream pornographic platforms. These tags are systematically deprecatory and offensive, using words such as 'whores', 'sluts', or verbs like 'punish', or 'bang'. In this manner, 'heteronormativity becomes the structural whole within which all pornographic material is understood.' (Saunders, 2020:87).

However, heteronormativity might also be further reaffirmed by the design of porn sites (Keilty 2018) and, in particular, by the increasingly ubiquitous role algorithms play in producing, distributing and curating content. The algorithms involved in pornography recognition and content moderation are argued to be very often biased towards specific forms of pornography, that recalls heteronormative standards and reproduces a male point of view (Gerrard \& Thornham 2020; van der Nagel 2013). Gehl and colleagues (2017) point out that these algorithms are largely created by computer scientists who inscribe "assumptions about pornography, human sexuality, and bodies" into their coding work; for them, they argue, "pornography is limited to images of naked women", and "sexuality is largely comprised of men looking at naked women"; accordingly, "pornographic bodies comport to specific, predictable shapes, textures, and sizes". As a result, they conclude, "computer scientists appear to be training computers to see the narrow form of pornography described above while dismissing a heterogeneous array of other forms of pornography (gay, 
queer, trans*, hardcore, fat, bondage, hairy, and so much more) as "noise."” (Gehl et al. 2017, 530)

Nonetheless, despite the increasing interest towards the relationship between algorithms and pornography, the role played by algorithmic content recommendation systems in reiterating heteronormative perspectives on gender and sexual interests has so far been somewhat overlooked. For these reasons, we deem timely to analyse the role played by algorithms and algorithmic personalization in the reiteration of a heteronormative porn culture on Pornhub.

\section{The platformization of porn}

Porn can and should be included among the many forms of cultural production that are subject to processes of platformization. As occurred in other contexts, such as the music (Bonini and Gandini 2019) and film (Lobato 2019) industry, also in the porn industry we are witnessing a reconfiguration of the forms of production, distribution, and monetization of content, that are now being subdued to platform logic (Duffy et al. 2019). Platform logic promotes the standardization of content based on criteria of popularity and predictability. Therefore, it becomes necessary to focus more closely on the specific ways in which pornographic content and the platform logic collide, and to question in particular how the algorithmic infrastructures that organize and distribute porn content relate to established notions of gender representation and sexual normativity.

Platform economies, research suggests, are built on the imperative to homogenize and render predictable the productive activity of viewers (Zuboff 2019); in this sense, the platform infrastructure represents the most efficient form of organization and circulation of content to maximise this logic (Nieborg and Poell. 2018). Unsurprisingly, porn content makes no exception; in this context, Pornhub has affirmed as the main gateway for the access to pornographic digital 
content. In the domain of porn, the 'monopolistic tendencies' (Srnicek 2017) that characterise platform capitalism are exemplified by Mindgeek, which owns Pornhub as well as many of the most popular porn sites (e.g., RedTube, YouPorn) and distributors (e.g. Reality Kings, Brazzers, see Lord, 2020). Property of Mindgeek, Pornhub incarnates the 'mainstreaming' of porn cultures; thanks to the popularity of Pornhub, pornographic content has grown mundane over the last decades, exiting the niche of 'dark' sexualised material to become ubiquitous in popular culture (Paasonen et al. 2019). Peculiar to the role that Pornhub holds in this context is an extensive PR campaign centered on the publication of yearly reports about video consumption on the platform - named 'Year in Review' - as well as of contextual data that identify trends in porn consumption through the platform on specific occasions, such as international soccer matches or other popular (local and global) events (Pornhub Insights 2021). These 'pop' reports and the fancy infographics these showcase contribute to fulfil the PR goal of being seen as a transparent company (Rodeschini 2021), that is also up to speed with the latest trends and innovations. At the same time, they shape the mainstream conversation around pornographic content and make its terminology and practices more accessible and appealing to everyday users. As argued by Paasonen et al. (2019), this is an attempt to rebrand porn consumption as an ordinary recreational activity and to define Pornhub as a 'stigma-free' environment. However, in their view (2019), this has the ultimate goal of transforming Pornhub into an advertising platform that exploits the data it collects about user behaviour on the platform to predict their activity and thus expose them to relevant ads, just like other social media platforms such as Facebook or YouTube. This is relevant, as the categorization of individuals typical of platforms, when applied to pornographic content, leans on sensitive information such as gender and sexual identities. Despite its obvious distinctive traits, at its core Pornhub is actually no different from any other platform (Macdonald 2019). As it maintains a 
'hands-free' approach towards its consumers and especially towards its content producers, essentially branding itself as a somewhat neutral facilitator of content distribution that offers content creators access to relevant audiences and claims little (if none at all) responsibility over the kind of content it hosts (Lord 2020). Like Netflix or YouTube, Pornhub is concerned with the necessity to maximise user information to the aim of offering targeted content to users. However, while Netflix or YouTube can rely upon access metadata as indicators of content value, and thus primarily reward permanence on the platform (Lobato 2016; Postigo 2016), Pornhub is instead in the business of distributing a type of content that is not typically consumed for long sessions. As a result, it is reasonable to assume it primarily rewards the return of users to the platform - not their permanence. Moreover, like YouTube it embeds an algorithmic recommendation system that presumably, since its exact formulation is not publicly known - suggests videos to users on the basis of a combination of computational and social logics (Airoldi et al. 2016) which demands specific attention. This becomes all the more pressing insofar as recommended video content on Pornhub intersects with existing gender norms,-sexual cultures, and identities, and thus might contribute to produce biased representations and reproduce heteronormative views of sexuality that counter the trend towards publics diversification.

\section{Methodology}

In order to consider Pornhub's personalization mechanisms it is first necessary to observe the Pornhub homepage and see how the specific categories of 'gender' and 'sexual orientation' are defined by the platform using fixed categories (see Figure 1). Almost a third of the daily visitors of the site are registered users, or over 22 million over 75 million (as of 2017, see Pornhub Insights 2021), as creating an account is the only way to access all the platform features such as commenting, liking, adding friends, subscribing or activating a premium account. Only logged- in 
users can explicitly specify their orientation and gender. Yet notably Pornhub never mentions gender or sexual orientation directly in the registration phase. Rather, they are respectively mentioned as ' $I$ am' and 'I like'; this is only true in this context, as they are called gender and interests (interested in, more specifically) on the account pages throughout the website after the registration. This is to some extent explained by the necessity of Pornhub to allow couples as a registration option, albeit it hardly being a gender. For clarity, in this paper we will refer to the gender specified during the registration phase as self-disclosed gender identity, and to interests as sexual interests. Aside from accounts and their variables, the focus will be on other affordances as well: categories, hyperlinks, layout and, in general, the technical infrastructure of the website.

\section{How do you like it?}

Tell us about yourself and what turns you on so we can provide you with the content that suits your tastes.

\section{I am a:}

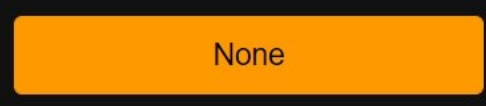

Couple

Trans Woman

\section{Non-Binary}

Male
Female

\section{Same Sex Couple (Male)}

I like:

Figure 1, Pornhub's signup categories

Data collection processes leveraged on the 'Pornhub Tracking Exposed' (poTREX) infrastructure (Agosti \& Corona 2020), a product of the free-software initiative tracking.exposed. Tracking 
Exposed aims to 'put a spotlight on users' profiling, on the data market and on the influence of algorithms' (tracking.exposed 2018), by collecting data from personalization algorithms (e.g. Hargreaves et al. 2018; Beraldo et al. 2021; Sanna et al. 2021). Specifically, poTREX consists of a browser extension that collects and processes data from Pornhub.com web pages such as page layout, video order, titles and views, authors, categories, and more. While poTREX's extension has been used as a data collection tool following a specific research design, it has also been freely available for aggregated and distributed data collection by voluntary users since 2019; furthermore, top-down coordinated testing took place in the beginning of 2020. This additional data has been useful to provide further glimpses into the evolution of the platform.

\section{Homepage overview}

To obtain an overview of the landing page, we accessed the homepage multiple times in a short time span: this proved necessary to reduce potential time-related sensitivity, as underlined during preliminary experiments (Agosti \& Corona 2020). This procedure has been repeated twice over a two-week span, with 600 homepages collected on the first run and 1000 on the second, with a 15seconds delay between each homepage access; these, as well as further data collections, originated from Italy - the authors' location - with the language of the platform set to English.

Through this process we retrieved 1600 homepages, with 46 videos per access: since we used unauthenticated accounts, Pornhub was serving a homepage layout with four sections (more on this below). The first collection lasted for two hours of intense periodic automated access, during which we retrieved data about 27.508 videos over a potential amount of 27.600 videos - 46 per homepage- achieving $99.66 \%$ reliability, of which 122 were unique videos. During the second access we were able to collect data about 45.959 videos (99,91\% reliability), with 118 unique 
videos. This data collection helped us to determine potential recurring patterns, especially regarding the underlying logics governing the different sections of the homepage. Based on this, we progressively isolated potential intervening variables: we tested using multiple accounts, different IP addresses, operating systems, web browsers (with and without user trackers); we also considered the ways in which platforms tend to install trackers and link users' history. All of this allowed us to isolate the variables leading to homepage differences, and to evaluate whether differences were due to localization, browsing patterns, or due to personalization. A recommendation is a decision taken upon an estimated user profile; companies might profile based on how users behave on their platform or by buying profiles from third-party trackers (Maris et al, 2019). We concluded that Pornhub bases profiling only on the data possessed by companies in the Mindgeek network, without external inputs: this allowed us to consider personalization processes as mostly affected by three variables: the IP address, used to determine country-specific sections such as "Most viewed videos in Italy" or "Hot porn videos in Italy"; the language of the operating system, which has an effect on Pornhub's localization as well, but not in content selection. Finally, self-disclosed gender identity, when present, seems to be the most relevant variable for what concerns personalization, as it has an effect on two sections and the content within it: "Recommended for You" and "Recommended Category for You".

Furthermore, previous experimental data collection provided us with historically and geographically sparse data about the website, its homepage, and video metadata. This data is unstructured and peer-sourced, as it has been collected by volunteers crowd-sourcing data through poTREX. While not suitable to draw substantive conclusions, it provided us with a useful point of reference in relation to the ever-changing nature of the platform and its geographical logics. 


\section{Homepage and personalization}

To delve deeper into the personalization processes of Pornhub around specific self-disclosed gender identities, we then collected a small number of homepages, observed through specific accounts. This approach situates itself in the methodological tradition of the reverse engineering of platform algorithms (Diakopoulos 2014; Kitchin 2016), which looks at "what data are fed into an algorithm and what output is produced" (Kitchin 2016,24). This constitutes a valuable strategy to account for the algorithmic relations among digital objects on a given platform, but also presents some significant methodological constraints. As Sophie Bishop (2018) notes, to rely on the inputoutput of data means to be dependent on what can (and cannot) materially be accounted for through it. This may - or may not - be sufficient for empirical research on algorithmic relations depending on the research question asked and on the amount of information available about the platform observed - which is often 'black-boxed' - and inevitably leaves some areas unscrutinised. Furthermore, this method is highly exposed to sudden changes in the platform architecture, which represents a significant risk during the course of the data collection. In fact, some changes to the platform did occur in the context of this research, as will be expanded upon below. Yet, we maintain this remains a highly effective pathway of inquiry as it offers a granular account of individual experiences of 'algorithmic situations', intended as the moment in which users encounter - and, to some degree, recognize and exploit - the intervention of an algorithm in their platform experience (Public Data Lab 2021).

In this case, the inputs were ad-hoc accounts created with specific socio-demographic characteristics, while the output was the content and the layout of the Pornhub's homepages shown to these accounts. We focused on the homepage as it is the most visible part of the website, and therefore common to most users regardless of individual browsing patterns. This meant collecting 
less videos when compared to pages offering only personalized content, but at the same time ensuring that those collected are in fact consistent and indicative of the platform experience by an average user. Furthermore, focusing on homepages allowed us to consider the bulk of recommended content that is not personalized (e.g., Most Viewed Videos).

The accounts (or sock puppets, see Sandvig et al. 2014) were created following the signup procedure on the platform: from a total of 30 possible combinations of self-disclosed gender identity (10) and sexual interests (3) we derived a total of 10 accounts, as detailed in the chart below (figure 2). Concerning accounts, all self-disclosed identities were included, while leaving sexual interests stable for girls. This is above all to avoid possible distortions given, for example, by the redirecting of some accounts interested in guys to the gay portion of the website. Secondly, because in this research we particularly aim to grasp how the personalization changes across selfdisclosed gender identities, which can more clearly emerge by leaving the second variable, sexual interest, fixed.

Other fields such as formal video characteristics (e.g., preferred video length or production type) or specific preferences (e.g., categories or body types) were left blank. We then collected the content and layout of every homepage visualized by each account every day for a week. The final corpus of data for our analysis is composed of ten accounts accessing the website for seven days; over the course of three minutes they automatically collected a snapshot of the Pornhub homepage, for a total of 490 times. As each access records 51 videos, we collected data about 24990 videos (including duplicates) between the 12th and the 17th of February.

Initially, the research included a second dataset, collected with the same procedure and variables but with new accounts, one month later. The inclusion of a second dataset was aimed at reducing 
any potential artefacts given by changes in the platform, as well as to enhance reliability. However, the second dataset coincided with structural changes to Pornhub's homepage layout, as will be expanded below, given by deep changes in algorithmic recommendation systems. Therefore, only the first data collection can reliably be included in our final corpus.

\begin{tabular}{|ll|}
\hline Sexual Interests & Self-Disclosed Gender Identity \\
\hline Girls & Couple \\
& Female \\
& Male \\
& Non-Binary \\
& None \\
& Other \\
& Same Sex Couple (Female) \\
& Same Sex Couple (Male) \\
& Trans Man \\
& Trans Woman \\
\hline
\end{tabular}

Figure 2, accounts and disclosed characteristics

Accounts were created and data was collected through ad-hoc browsing environments, with browser cookies isolated between accounts. It is relevant to note how the platform might identify its users through other means, such as hardware configuration or advertising ID (Surveillance Self-Defense 2020). However, due to the nature of the research design, personalization is still expected to vary in a consistent and comparable way across accounts. 
To consider personalized content we mostly leaned on a comparative approach between accounts. To this end, we used social network analysis and related visualisation techniques, thus considering accounts and videos as nodes in a bipartite network and recommendations as edges: every time a account visualized a video on its homepage, the video and the account were connected. This allowed us to graphically render the degree to which accounts were recommended personalized or blanket content, and whether these recommendations created clusters among self-disclosed identities. For the latter, modularity has been calculated using Louvain algorithm (Blondel et al. 2008) through Gephi (Bastian et al. 2009). This algorithm creates recommendation clusters, by grouping together videos that are that are suggested to similar accounts. We used Circular Layout to visually force profiles nodes to a circular position and ForceAtlas2 (Jacomy et al. 2014) to place recommended video nodes according to their relation with accounts.

\section{Findings}

\section{Homepage overview}

The layout of PornHub's homepage is composed of different sections, each collecting the previews of several videos. Through our data collection, and supported by exploratory data from poTREX (poTREX 2020), the standard layout composes five sections, in the following order (from top to bottom): Hot Porn Videos in Your Country; Most Viewed Videos in Your Country; Recommended for you; Recommended category for you; Recently Featured XXX Videos. Considering the median views per section (see figure 3), it appears that Pornhub's groupings are aptly named: the 'Most Viewed' videos had by a large margin the highest number of visualizations $(2.5 \mathrm{M})$, followed by 
hot videos $(1.3 \mathrm{M})$, recommended videos $(1.1 \mathrm{M})$, recommended categories $(159 \mathrm{k})$, and recently featured videos $(35 \mathrm{k}){ }^{1}$

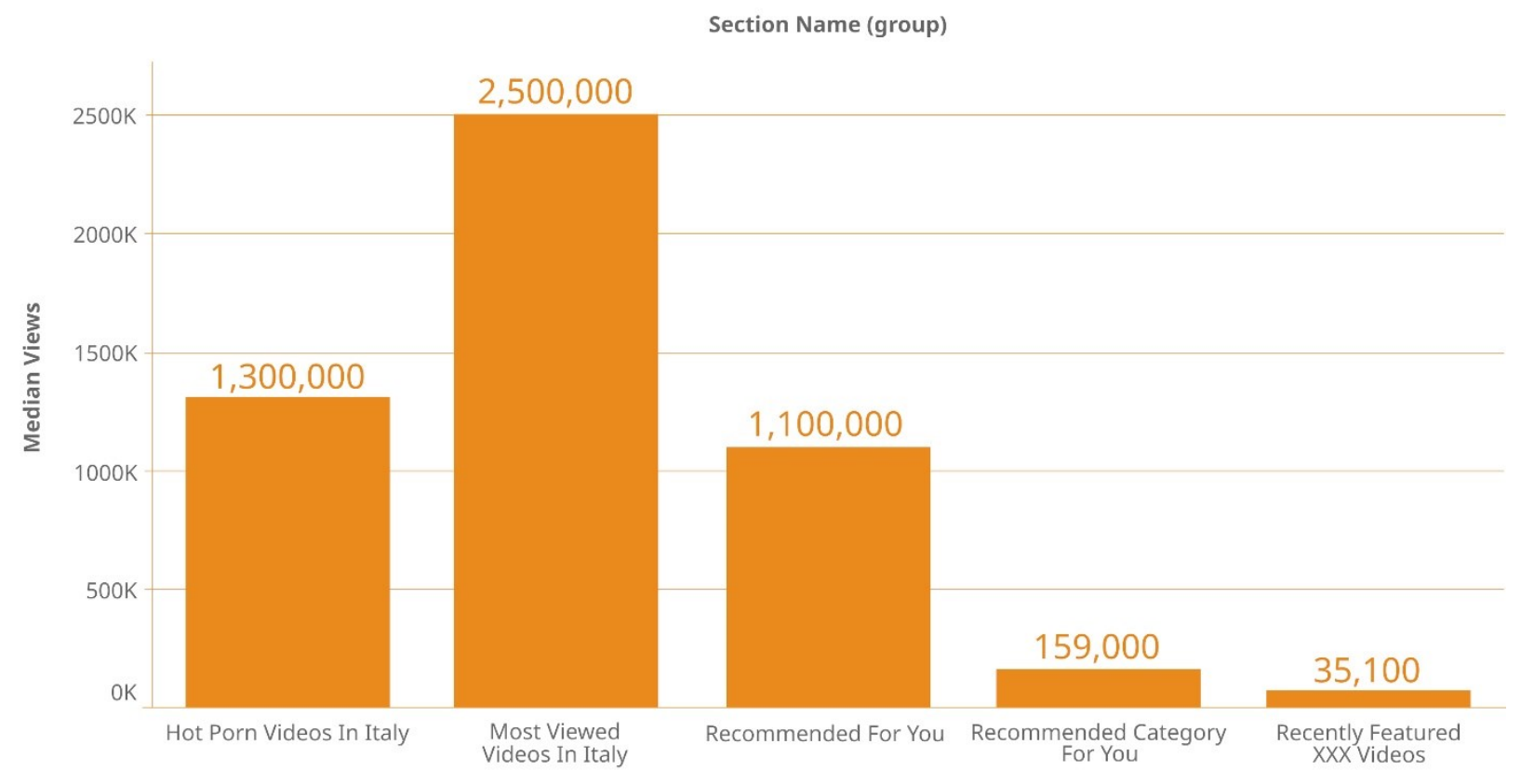

Figure 3, median views per section

The first two sections are based on the localization of users; in our case, therefore, the first two sections were Hot Porn Videos in Italy and Most Viewed Videos in Italy. Aside from a broader geographical personalization of content, an area deserving further inquiry, the prominence of these categories raises broader concerns about the cultural power of popularity measures to sort content. Similar to what van der Nagel (2013) suggests in her discussion of reddit's 'gonewild' pictures, data suggest that also on Pornhub the recommendation of sexual content following popularity criteria contributes to promote the circulation of supposedly 'ideal' views of sexuality, gender identities and body representations that leave little room for diversification (van der Nagel 2013). Overall, this produces 'ranking cultures' that reward certain types of content upon others on the

\footnotetext{
${ }^{1}$ Median views were calculated using data from the accounts included in the analysis.
} 
basis of visibility metrics, as is the case for YouTube (Rieder et al. 2018), thus enabling a kind of Matthew effect, a cumulative effect of accumulation whereby the rich (in visualizations) get richer, and the poor get poorer.

Beyond these standardised sections, the bulk of personalized content is in Recommended Videos for You, which presents a series of recommended videos, and in Recommended Category for You, that showcases a set of videos from a specific, personalized, category drawn from the categories of the website (e.g., amateur, asian, bondage, gangbang, MILF, teen). These sections are not set in stone, and over the years the Recommended Category for You section got progressively tuned down: historical evidence from poTREX (poTREX 2020) underlines how it existed for at least 2 years, up until the end of February 2021, when it got removed. ${ }^{2}$ This mutability to some extent applies to concurrent instances of the website as well: accessing from certain countries returns a language-specific section (e.g., Videos in German language). While the homepage is relatively standardised across time and across countries, these variations are nonetheless noteworthy, as they point to some degree of geographical specificity concerning content recommendation on the platform.

In order to keep their user base active and engaged, digital platforms tend to shift rapidly, with frequent structural changes in layout, affordances, scope, and features (Bucher \& Helmond 2018). This typically serves the main purpose of an accurate and granular gathering of data about user behaviour and activity on the platform, which in turn represents the baseline for its own economic existence (Zuboff, 2019; Srnicek, 2017). Our data illustrate that PornHub is no exception to this

\footnotetext{
${ }^{2}$ For some registered users it has been replaced by a New Videos From Your Subscriptions section, composed by the new videos from channels and verified users, as based on subscription to these content creators.
} 
logic. During the writing of this article, the platform altered how it manages the distribution of user-generated content (i.e., the removal of all content from unverified users in December 2020, previously amounting to around $25 \%$ of homepage videos), and enforced changes in the homepage layout. This confirms the degree of similarity that exists between Pornhub and other 'traditional' platforms, in particular YouTube (see also Arthurs et al., 2018), in the attempt to optimize the relationship between algorithmic recommendation of content and user preferences for the ultimate purpose of data gathering and user profiling. Yet, the application of these logics of optimization and fine-tuning of content recommendation entangles with the very specific type of content circulating on the Pornhub platform, which raises the question of whether and to what extent these processes foster the circulation of content that reinforces heteronormative viewpoints and sexual practices.

\section{Profiles, heteronormativity, and Pornhub as platform(s)}

The construction of accounts on social network sites and digital platforms is relevant, both for consumers and producers (Bruns 2007). How platforms conceptualise gender also has broader effects, as it determines a specific, socially embedded cultural conception that is able to shape, affect, and maintain gender identities (Bivens et al. 2016). Considering how online pornography affects gender exploration and performativity (Scarcelli 2015), the role of websites such as Pornhub is considerable in this regard, especially when contextualised in a digital pornographic environment that is becoming increasingly social (Tyson et al. 2015; Drenten et al. 2020; Wang 2021). Social media such as Instagram and Twitter are used to promote content on websites such 
as Pornhub and OnlyFans, which in turn allow for the creation of accounts and promote interaction among users.

To investigate how the algorithmic recommendation system on the Pornhub platform might reiterate a heteronormative point of view, we created several accounts with the intention to investigate differences in recommended and personalized content. In our research, the user accounts we created were assigned a variable gender identity and a fixed sexual interest during the registration phase. While creating the accounts, however, signs of a binary understanding of sexual orientation were already reflected through Pornhub's affordances. The creation process invites users to specify a self-disclosed gender identity from a section called ' $I a m a^{\prime}$ ', as shown in figure 1, from several choices: None, Male, Female, Couple, Same Sex Couple (Female), Same Sex Couple (Male), Trans Woman, Trans Man, Other, Non-binary. Sexual interests, presented as 'I like', are instead collapsed into three categories: Girls, Guys, Guys and Girls. Based on these values, the rest of the web page dynamically changes to inquire into the taste of the user, who is invited to specify some preferences among which are body characteristics such as ethnicity, hair color, and age, as well as preferred categories and channels. Notably, channels on Pornhub are how production houses distribute, promote, and monetize their content on the website, rather than being a synonym of public account.

The ways in which the sections of the registration page react to these choices vary. Concerning body type ('What do you like about girls/boys?'), this clearly reflects the sexual interests it receives as an input: if interested in girls, the user can then specify its preferred ethnicities and body features pertaining to women; similarly, if interested in Guys, the user is prompted to choose its favourite 
features concerning males. Categories and channels, however, change differently: specifying Guys or Girls as a sexual interest does not warrant a change in the content proposed in these sections on its own; male-oriented, heteronormative content is assumed to be the norm regardless of stated interests, up until the combination of self-disclosed gender identity and sexual interests matches how the platform preconceives male homosexual relationships. The switch to categories and channels catering to homosexual males takes place only if the sexual interests is set to Guys, and the self-disclosed identity is either Male, Same Sex Couples (Male), or Trans Male. This is not simply the way through which PornHub decides which categories to show a user during the registration phase, but also the way in which the platform can segment content and categorise individuals, in this case redirecting the user to PornHub Gay, a section of the platform dedicated to male homosexual content. This section presents roughly the same features of the main homepage for what concerns ranking and categorisations but has a less sophisticated homepage layout. This 'website within the website', while still retaining the same domain, underlines the interplay between the technical structure of the website, its affordances, and the specific social conceptions of sexual identity embedded within the platform. It is reflected in the segmentation of content into two separate flows, as it is not possible to access heterosexual videos from the homosexual section and vice versa. Aside from the separation of content into different parallel homepages, Pornhub and Pornhub Gay, the ways in which these portals can be accessed or left is relevant. While Pornhub is the standard homepage, Pornhub Gay is intended as a sub-section of the 'main' website: this is further exemplified by how affordances allow to switch from one section of the website to another. To get to homosexual content from Pornhub, the link is nested in the dropdown menu of the 'categories' section; conversely, to pass from the homosexual to the heterosexual platform, the 
link is clearly visible: the 'home' button from the 'standard' website is substituted by 'straight' on PornHub Gay.

However, this difference in categories and sections of the website does not cater to homosexual interests involving girls, trans identities, or to more fluid forms of sexuality. Overall, these findings suggests that categories on Pornhub are built with a heterosexual logic in mind; even when considering homosexual content, this is mainly intended for male homosexual content: the categories for a male interested in males, for example, display male homosexual intercourse, while the categories for a female interested in females display heterosexual intercourse - the same occurs while creating male into females accounts.

At a surface level, the discrepancies between possible self-disclosed identity and sexual interests are striking, especially given the nature of the website: a pornographic website could be expected to prioritize sexual interests over identity, as more pertinent to the type of content proposed. However, what the PornHub interface labels as gender is given considerably more weight than what is declared as sexual interests. This is exemplified by a recommending system that is tailored according to self-disclosed identity but never to sexual interests. But how does this fit into a platform that prioritizes sexual satisfaction? It might be possible to understand this decision as broadly nested into the political economy of the platform (and of platforms, see Bivens 2015), leveraging on LGBT+-positive attitudes for marketing and visibility purposes. The latter might also be related to the relevance given to the number of female visitors of the platform, a staple of the data porn reports released by Pornhub: Pornhub insights. In its yearly review, Pornhub declared a share of $24 \%$ of female visitors in 2014 , growing every year up to $32 \%$ in 2019 . No 
yearly insights were released in 2020 (Pornhub Insights 2021). The release of these analyses starts in 2013 (Pornhub Insights 2021). In the same year, Pornhub's parent company changes its name from Manwin to MindGeek (AVN 2013). The multiplicity of options might be an effort to profile users to provide personalized content: if this is the case, however, it might point to a greater relevance, data-wise, of self-declared gender identity as opposed to sexual interests in this regard.

\section{Homepage and personalization}

We then set out to consider the differences between homepages and recommended content based on self-disclosed gender identities. The first discrepancies appear when considering page layout: not all 10 accounts shared the same 5 sections (as detailed above), but Same Sex Couple (female), Non-Binary, Trans Female, and Trans Male, only had 4; this affected the homepage only when the account has 'sexual interest' set as female. When interested in males, or both, this difference was not present. The missing section is the Recommended Category for You, which is in turn relevant as it consisted in one of our two entry points into the personalized content shown on the homepage. This is because not all of Pornhub's homepage consists of personalized content: in fact, the majority of the sections recommend the same videos to all users, regardless of disclosed characteristics and with no discernible pattern. This is the case for the Hot Porn Videos in Your Country; Most Viewed Videos in Your Country; and the Recently Featured XXX Videos (figure 4). This allows us to conclude that personalization does not seem to affect the homepage at large: to continue our investigation, we then proceeded with considering sections individually. 


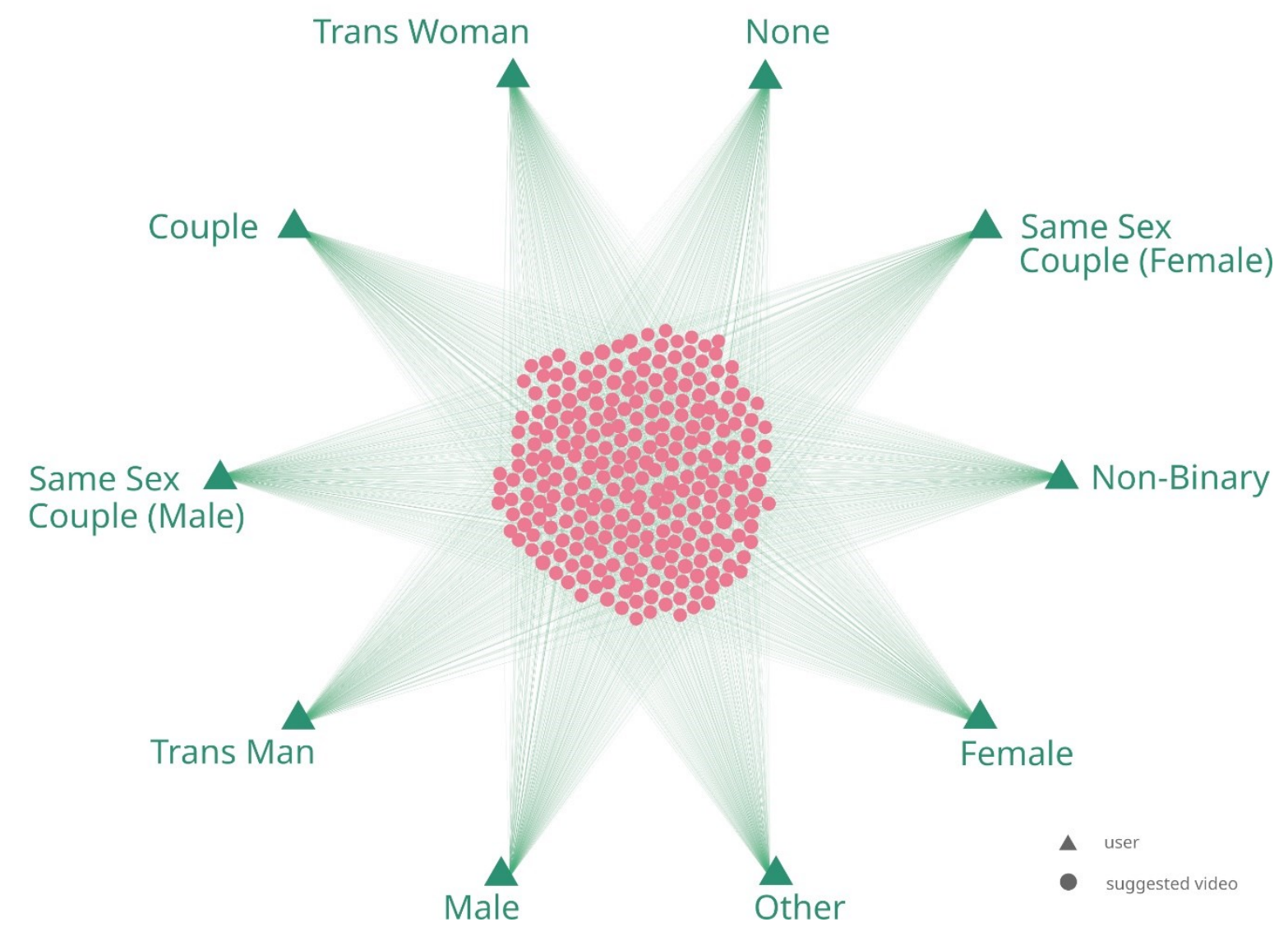

Figure 4, Videos suggested in the common sections (Hot Porn Videos in Your Country, Most Viewed Videos in Your Country, and Recently Featured XXX Videos).

Specifically, personalized content is concentrated in two sections: Recommended Videos for You and Recommended Category for You. The recommended category section is composed by several videos all pertaining to a specific category: this makes it possible to compare common content across accounts (figure 5). As different colors represent different clusters, some commonalities emerge: between Male and Female, and between Couple and Same Sex Couple (Male); conversely, None and Other have little content in common with any other account. This does not mean that accounts (the triangular nodes) of different clusters (colors) have no videos (the small, circular, nodes) in common with each other, but rather that this happens to be somehow not consistent 
enough to group them together. The pairings are relevant, as they indicate some form of gendernormativity in recommended video content: males and females, and heterosexual couples are grouped, while non-conforming genders have 'their own' specific recommendations. This is especially relevant when considering that this section is missing altogether for genders such as Same Sex Couple (female), Non-Binary, Trans Female, and Trans Male.

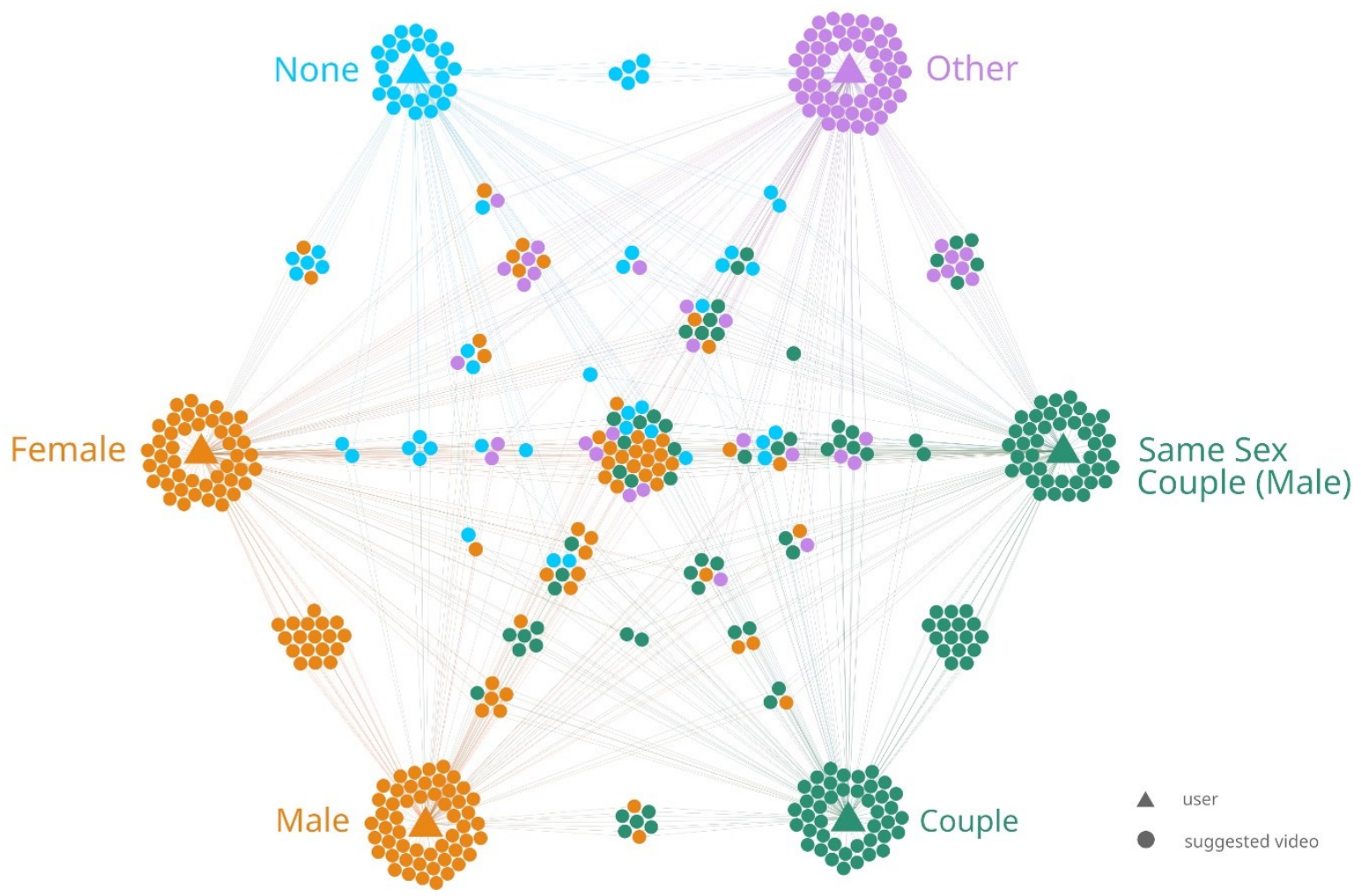

Figure 5, videos in Recommended Category for You based on account

This evidence suggests a gendered understanding of content by Pornhub, which is further reflected in the personalized category common to all accounts: Recommended for You (figure 6). When compared to the previous category the distinction is clearer: what were two separate clusters based on recommended videos now become one, comprised of Male, Female, Same Sex Couple (Male), 
Couple, with the addition of Other, which was previously a third distinct cluster. These accounts not only have the same content recommended to them (the group of blue nodes between the users), but aside from minor exceptions, they are recommended only this type of content. This is relevant as some of the users reflecting non-conforming gender identities (Trans Man, Trans Woman, Same Sex Couple (Woman), Non-Binary), aside from receiving shared personalized content among them, also receive personalize content exclusive to that gender (e.g., a Trans Woman account is recommended some videos that were not shown to any other account, as represented by the orange nodes surrounding the corresponding account). The account with gender set to None is the only one not adhering to these two sharply defined groups, and it mostly has videos recommended exclusively to it.

This underlines the role of affordances in managing different identities: once those are arbitrarily determined by Pornhub through the registration process, as shown above, content is then algorithmically segmented according to the specific, socially embedded, point of view of the platform. Algorithms and affordances such as accounts, settings, categories, and hyperlinks, therefore, contribute to foster this point of view. 


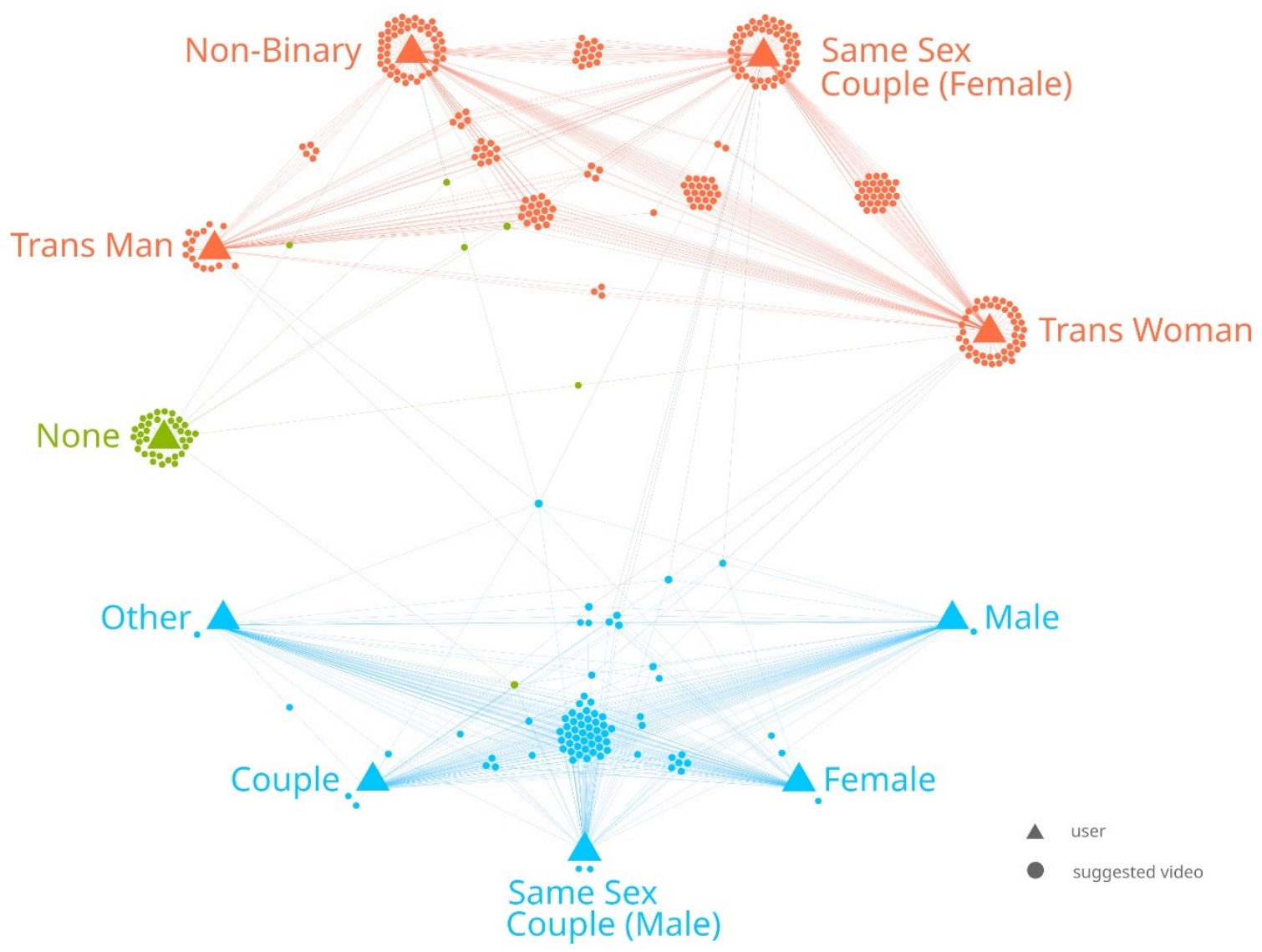

Figure 6, videos in Recommended for You based on account

The observed division is also confirmed on another hidden segmentation operated by Pornhub. By considering the video recommended for the different accounts, we examined the nature of the producer, intended as who uploaded the content. Among the three kinds of producer supported by the platform, which are model, channels, and pornstar, the gender-normative group presents recommended videos from all three kinds, while the second group does not include any content from channels. Considering that channels are mostly dedicated to production companies, this might also suggest that Pornhub manages content recommendation in relation to self-declared 
gender identity not only considering its algorithmic or personalization system, but factoring in broader productive and distributive logics as well.

While these results are consistent across dimensions and sections, it is necessary to underline the limits inherent to conflating gender as a concept and self-declared gender identity as a restricted choice in the registration phase. This is particularly reflected in the options Couples and None, that are not as neatly nested into broader groupings as other options. However, this provides a further look into how Pornhub decides to construct gender through its affordances, and how it broader recommendation and personalization patterns based on its understanding of gender and sexuality.

\section{Conclusion}

This article has presented empirical evidence on the specific ways in which Pornhub concurs to reiterate heteronormative perspectives on gender and sexuality through its platform affordances and algorithmic recommendation system. As shown, this happens on several levels throughout the platform, from the registration phase to the recommendation and personalization of content. The registration phase is where Pornhub establishes the identity of users by constructing their gender, and manages the distribution of content based on self-declared gender identity; the procedure presents a restricted and arbitrary choice, which guides and informs the videos recommended to the user. This significantly contributes to foster a heteronormative perspective by separating or undersizing unconforming content, for example by redirecting male homosexual users to an isolated portion of the website or structurally altering the homepage's layout for specific selfdeclared gender identities. 
Focusing on Pornhub's personalization and recommendation system, we have shown how this reflects gender-normativity by grouping and managing content based on conformity to hegemonic gender identities. This is reflected through the personalized suggestions: if a user is identified as a male, a female, a heterosexual couple, or a male homosexual couple, the recommended content is constant throughout all groups; conversely, it is shown different, isolated content - regardless of its declared sexual interest. Despite this granular personalization, the most visible content on the platform remains fixed and generally follows popularity logics. The combination of these insights shows how Pornhub, and pornographic platforms in general, might contribute to foster heteronormative perspectives on gender identity, sexual interests, and practices, to the potential detriment of minoritarian views, as a result of their infrastructural features and the decision that underpin their construction and maintenance.

It is worth noting that how Pornhub operationalizes gender through fixed categories suggests the broader economic need Pornhub has to define its users. While this has relevant implications, as shown, in terms of recommended content, it might also produce some distortions, for example in the use of somewhat blurred identities (e.g.: 'none' could be intended both as a category intended to adhere to gender, as well as a residual group, such as 'null'). Furthermore, accounts created with no data aside from self-declared gender identity and declared interests might not represent any real users; considering a similar limit related to browsing patterns, other methodological approaches beyond the one employed here might also prove useful. For instance, one that is more embedded into user practices (e.g., through personas) and leaning into more qualitative methodologies might lead to different (and equally interesting) results. 
Aside from methodological variations, and despite the limitations of our study, we contend that further research is needed to investigate in depth the algorithmic logics that are peculiar to a broader array of pornographic websites. The way these platforms profile users through undisclosed means, such as location, and how this might lead to the propagation of specific sexual practices through less apparent means, is pivotal to unearth the impact that digital platforms have in dictating and fostering hegemonic forms of sexuality and gender identity.

\section{Disclosure statement}

No potential conflict of interest was reported by the authors.

\section{Data availability statement}

The data that concur to support the findings of this study are openly available on Github at [https://github.com/tracking-exposed/experiments-data/tree/master/potests/potest_12-19feb].

\section{References}

Adult Video News. 2013. 'Manwin Becomes MindGeek'. 8 October. Adult Video News Accessed January 6 2022. https://avn.com/business/articles/technology/manwin-becomesmindgeek-534744.html.

Agosti, Claudio and Giulia Corona. 2020. 'PornHub Tracking Exposed' 1 January. Accessed January 6 2022. https://pornhub.tracking.exposed/. 
Albury, Kath. 2014. 'Porn and sex education, porn as sex education'. Porn Studies 1 (1-2): 172181. https://doi.org/10.1080/23268743.2013.863654

Arvidsson, Adam. 2007. 'Netporn: the work of fantasy in the information society' In: C'Lick Me: A Netporn Studies Reader, edited by Katrien Jacobs, Marije Janssen, Matteo Pasquinelli, 6976. Amsterdam: Institute of Network Cultures.

Arthurs, Jane, Sophia Drakopoulou and Gandini, Alessandro. 2018. 'Researching YouTube'. Convergence 24(1): 3-15. https://doi.org/10.1177/1354856517737222

Ashton, Sarah., Karalyn McDonald and Maggie Kirkman. 2019. 'What Does 'pornography' Mean in the Digital Age? Revisiting a Definition for Social Science Researchers'. Porn Studies, 6(2): 144-168. https://doi.org/10.1080/23268743.2018.1544096.

Barrie, Joshua. 2017. 'This is how much of the internet is porn'. 9 February. Mirror. Accessed January 6 2022. https://www.mirror.co.uk/tech/how-much-internet-porn-9784557.

Bastian, Mathieu, Sebastien Heymann, and Mathieu Jacomy. 2009. 'Gephi: An Open Source Software for Exploring and Manipulating Networks'. Proceedings of the International AAAI Conference on Web and Social Media 3 (1):361-62.

https://ojs.aaai.org/index.php/ICWSM/article/view/13937. Bishop, Sophie. 2018. 'Anxiety, panic and self-optimization: Inequalities and the YouTube algorithm'. Convergence 24(1): 69-84.

Beraldo, Davide, Stefania Milan, Jeroen de Vos, Claudio Agosti, Bruno Nadalic Sotic, Rens Vliegenthart, Sanne Kruikemeier, Lukas P. Otto, Susan A.M. Vermeer, Xiaotong Chu and Fabio Votta. Political advertising exposed: Tracking Facebook ads in the 2021 Dutch elections. 
Internet Policy Review. https://policyreview.info/articles/news/political-advertising-exposedtracking-facebook-ads-2021-dutch-elections/1543

Bivens, Rena. 2017. 'The gender binary will not be deprogrammed: Ten years of coding gender on Facebook'. New Media \& Society 19(6): 880-898.

https://doi.org/10.1177/1461444815621527

Bivens, Rena and Olivier L. Haimson. 2016. Baking Gender Into Social Media Design: How Platforms Shape Categories for Users and Advertisers. Social Media + Society. https://doi.org/10.1177/2056305116672486

Blondel, Vincent D., Jean-Loup Guillaume, Renaud Lambiotte and Etienne Lefebvre. 2008. Fast unfolding of communities in large networks. Journal of Statistical Mechanics: Theory and Experiment.

Bonini, Tiziano and Alessandro Gandini. 2019. First week is editorial, second week is algorithmic": Platform gatekeepers and the platformization of music curation. Social Media + Society.

Bruns, Axel. 2009. 'From Prosumer to Produser: Understanding User-Led Content Creation'. September 3. Transforming Audiences 2009. England, London.

Bucher, Taina. 2012. 'Want to be on the top? Algorithmic power and the threat of invisibility on Facebook'. New Media \& Society 14(7): 1164-1180. https://doi.org/10.1177/1461444812440159

Burke, Nathaniel B.. 2016. 'Straight-acting: Gay pornography, heterosexuality, and hegemonic masculinity'. Porn Studies 3(3): 238-254. https://doi.org/10.1080/23268743.2016.1196117 
Diakopoulos, Nicholas. 2014. "Algorithmic Accountability Reporting: On the Investigation of Black Boxes”. Tow Center for Digital Journalism, Columbia University. https://doi.org/10.7916/D8ZK5TW2

van Dijck, José, Thomas Poell and Martijn de Waal. 2018. The Platform Society: Public Values in a Connective World. Oxford University Press.

Drenten, Jenna, Lauren Gurrieri and Meagan Tyler. 2020. 'Sexualized labour in digital culture: Instagram influencers, porn chic and the monetization of attention'. Gender, Work \& Organization 27(1): 41-66. https://doi.org/10.1111/gwao.12354

Duffy, Brooke Erin, Thomas Poell, David B. Nieborg. 2019. Platform Practices in the Cultural Industries: Creativity, Labor, and Citizenship. Social Media + Society.

Electronic Frontier Foundation. 2014. “About Surveillance Self-Defense”. May 8. Accessed January 6 2022. https://ssd.eff.org/en/about-surveillance-self-defense.

Fritz, Niki, Vinny Malic, Paul Bryant and Yanyan Zhou. 2020. Worse Than Objects: The Depiction of Black Women and Men and Their Sexual Relationship in Pornography. Gender Issues. https://doi.org/10.1007/s12147-020-09255-2

Gehl, Robert W., Lucas Moyer-Horner and Sarah K. Yeo. 2017. 'Training Computers to See Internet Pornography: Gender and Sexual Discrimination in Computer Vision Science'. Television \& New Media 18(6):529-47. https://doi.org/10.1177/1527476416680453. 
Gerrard, Ysabel and Helen Thornham. 2020. 'Content moderation: Social media's sexist assemblages'. New Media and Society 22(7): 1266-1286. https://doi.org/10.1177/1461444820912540.

Hargreaves, Eduardo, Claudio Agosti, Daniel Menasché, Giovanni Neglia, Alexander ReiffersMasson and Eitan Altman. 2018. Biases in the Facebook News Feed: A Case Study on the Italian Elections. 2018 IEEE/ACM International Conference on Advances in Social Networks Analysis and Mining (ASONAM). https://doi.org/10.1109/ASONAM.2018.8508659

Jacomy, Mathieu, Tommaso Venturini, Sebastien Heymann and Mathieu Bastian. 2014. 'ForceAtlas2, a Continuous Graph Layout Algorithm for Handy Network Visualization Designed for the Gephi Software'. PLoS ONE 9(6): e98679. https://doi.org/10.1371/journal.pone.0098679

Keilty, Patrick. 2018. 'Desire by Design: Pornography as Technology Industry'. Porn Studies 5(3):338-342. https://doi.org/10.1080/23268743.2018.1483208.

Kitchin, Rob. 2016. 'Thinking critically about and researching algorithms'. Information, Communication \& Society 20(1): 14-29. https://doi.org/10.1080/1369118X.2016.1154087.

Lobato, Ramon. 2019. Netflix nations: The geography of digital distribution. New York: NYU Press.

Lobato, Ramon. 2016. 'The cultural logic of digital intermediaries: YouTube multichannel networks'. Convergence 22(4): 348-360. https://doi.org/10.1177/1354856516641628.

Lord, Phil. 2020. 'Pornhub: Opening the Floodgates?'. Houston Law Review Off the Record 54, 11(2):54-59. http://dx.doi.org/10.2139/ssrn.3751640. 
MacDonald, Margaret. 2019. Desire for Data: PornHub and the Platformization of a Culture Industry (Doctoral dissertation, Concordia University).

Maris, Elena, Timothy Libert, and Jennifer R. Henrichsen. 2020. 'Tracking sex: The implications of widespread sexual data leakage and tracking on porn websites'. New Media \& Society 22(11):2018-2038. https://doi.org/10.1177/1461444820924632.

Mazières, Antoine, Mathieu Trachman, Jean-Philippe Cointet, Baptiste Coulmont and Cristophe Prieur. 2014. 'Deep Tags: Toward a Quantitative Analysis of Online Pornography'. Porn Studies, 1(1-2):80-95. https://www.tandfonline.com/doi/abs/10.1080/23268743.2014.888214. van der Nagel, Emily. 2020. 'Verifying Images: Deepfakes, Control, and Consent'. Porn Studies 7(4):424-429. https://doi.org/10.1080/23268743.2020.1741434.

van der Nagel, Emily. 2013. Faceless Bodies: Negotiating Technological and Cultural Codes on reddit gonewild. Journal of Media Arts Culture. http://scan.net.au/scn/journal/vol10number2/Emily-van-der-Nagel.html.

Nieborg, David B. and Thomas Poell. 2018. 'The platformization of cultural production: Theorizing the contingent cultural commodity'. New Media \& Society 20(11): 4275-4292.

Noble, Safiya Umoja. 2018. Algorithms of Oppression: How Search Engines Reinforce Racism. New York: NYU Press. https://doi.org/10.2307/j.ctt1pwt9w5

Paasonen, Susanna. 2009. 'Online Pornography, Normativity and the Nordic Context'. In: Cyberfeminism in northern lights: Digital media and gender in a nordic context, edited by Malin Sveningsson Elm and Jenny Sundén, 51-72. Cambridge Scholars Publishing. 
Paasonen, Susanna, Kylie Jarrett and Ben Light. 2019. NSFW: sex, humor, and risk in social media. Cambridge: MIT Press.

Pasquale, Frank. 2016. The Black Box Society. Cambridge: Harvard University Press.

Pornhub. 2021. "Pornhub Insights". Accessed January 62022.

https://www.pornhub.com/insights/

Postigo, Hector. 2016. 'The socio-technical architecture of digital labor: Converting play into YouTube money'. New Media \& Society 18(2): 332-349.

Public Data Lab. 2021. “A Field Guide to Algorithms”. Accessed January 62022. https://publicdatalab.org/projects/a-field-guide-to-algorithms/ (forthcoming).

Raustiala, Kal and Cristopher Jon Sprigman. 2018. The Second Digital Disruption: Streaming \& the Dawn of Data-Driven Creativity. New York University Law Review 94(6): 18-30.

Ritzer, George and Nathan Jurgenson. 2010. 'Production, Consumption, Prosumption: The nature of capitalism in the age of the digital 'prosumer'. Journal of Consumer Culture 10(1): 13-36. https://doi.org/10.1177/1469540509354673

Rodeschini, Silvia. 2021. 'New standards of respectability in contemporary pornography: Pornhub's corporate communication'. Porn Studies 8(1): 76-91.

Sandvig, Christian, Kevin Hamilton, Karrie Karahalios and Cedric Langbort. 2014. "Auditing Algorithms: Research Methods for Detecting Discrimination on Internet Platforms". May 22. Data And Discrimination: Converting Critical Concerns Into Productive Inquiry, A 
Preconference At The 64th Annual Meeting Of The International Communication Association. Seattle, WA, USA.

Sanna, Leonardo, Salvatore Romano, Giulia Corona, Claudio Agosti. 2020. YTTREX: crowdsourced analysis of YouTube's recommender system during COVID-19 pandemic. Information Management and Big Data. https://www.springerprofessional.de/en/yttrexcrowdsourced-analysis-of-youtube-s-recommender-system-dur/19155456

Saunders, Rebecca. 2020. Bodies of Work: The Labour of Sex in the Digital Age. New York: Springer International Publishing.

Scarcelli, Cosimo Marco. 2015. ' It is disgusting, but ... ': Adolescent girls' relationship to internet pornography as gender performance'. Porn Studies 2(2-3): 237-249.

https://doi.org/10.1080/23268743.2015.1051914

Tyson, Gareth, Yehia Elkhatib, Nishanth Sastry, and Steve Uhlig. 2021. "Are People Really Social in Porn 2.0?". Proceedings of the International AAAI Conference on Web and Social Media 9 (1):436-44. https://ojs.aaai.org/index.php/ICWSM/article/view/14601. Tracking Exposed. 2021. “Tracking Exposed Manifesto”. Accessed January 62022.

https://tracking.exposed/manifesto/

Wang, Yidong. 2021. 'The twink next door, who also does porn: Networked intimacy in gay porn performers' self-presentation on social media'. Porn Studies, 8(2), 224-238. https://doi.org/10.1080/23268743.2020.1841019

Zuboff, S. (2019). The age of surveillance capitalism: The fight for a human future at the new frontier of power. New York: Profile books. 
